\title{
An Activity-Centered Wearable Computing Infrastructure for Intelligent Environment Applications
}

\author{
Dipak Surie and Thomas Pederson \\ Department of Computing Science, Umeå University, \\ S-901 87 Umeå, Sweden \\ \{dipak, top\}@cs.umu.se
}

\begin{abstract}
There are many research efforts that focus on converting everyday environments into intelligent and computationally active environments that support and enhance the abilities of its occupants in executing their activities. Such environments must have the ability to recognize the activities performed by its occupant, maintain a real-time model of the environment, address the occupant's privacy and personalization issues, and provide interaction capabilities in a way the occupant would with other people. In this paper we present an activity-centered wearable computing infrastructure for designing intelligent environment applications based on the occupant's usage and storage of everyday objects in that environment. Four components namely object manager, situative space monitor, activity recognizer and egocentric interaction manager are introduced and described in detail. A prototypical intelligent home environment capable of supporting 15 Activities of Daily Living with an activity recognition precision of $92 \%$ is presented as a "proof of concept" in a virtual reality (VR) simulated home environment.
\end{abstract}

\section{Introduction}

There are many research efforts that focus on converting everyday environments into intelligent and computationally active environments that support and enhance the abilities of its occupants in executing their activities [1], [2], [3]. Such environments must have the ability to detect its current state, the current state of its occupant and the activity performed by the occupant for providing computational support that are typically different to the office activities currently supported by desktop computing. Examples of applications that are developed for intelligent environments include providing support for activities of daily living at homes [4], for surgical activities in operation theatres, for mechanical activities in car workshops, etc. A major problem in building such applications has been the lack of uniform support for modelling and detecting: 1) the occupant's activities and actions; 2) the occupant's interactive status in terms of situative and multi-modal access to input and output devices; and 3) the occupant's environmental status in terms of available resources and their states. Many efforts in developing intelligent environments are heavily influenced by the underlying technology resulting in a lack of generality in developing such environments. We believe that starting out from a perspective centred around how occupants literally 
perceive the environment, based on the occupant's usage and storage of everyday objects in that environment and based on the weight that current cognitive science give to activity-driving factors could offer a valuable complement. In particular, it could offer a conceptual design platform robust enough to survive and handle generations of changes in the field of sensor technology.

Intelligent environments are public and private spaces that allow computers to participate in everyday activities that have previously never involved computation and allow occupants to interact with computers in a way they would with other people: via gesture, voice, context, etc. [5]. We follow similar design goals in developing an activity-centered wearable computing infrastructure for an intelligent home environment application capable of providing support for patients suffering from mild-dementia in performing their Activities of Daily Living (ADL) [4]. Four components namely object manager, situative space monitor, activity recognizer and egocentric interaction manager are introduced and described in detail.

\section{Challenges Involved in Designing Intelligent Environments}

Designing intelligent environments involves many challenges of which we address the following four fundamental challenges.

\subsection{Activity Recognition}

Intelligent environments that provide support for the occupant's activities should understand the concept of an activity and provide provisions for handling it [6]. Activity recognition in everyday environments is difficult due to the number, variety and variations in activities performed by the occupants ${ }^{1}$. For many intelligent environment applications, knowing the inhabitant's activity information alone is not sufficient [4]. This introduces a necessity to recognize activities and actions related to those activities with fine granularity; to recognize those activities during the initial phase of an activity before the activity reaches an irreversible state; and to determine the end of an activity [7], [8]. According to Activity Theory [9], human activities have an objective and are mediated through tools. We consider the objects present in the occupant's environment as tools for accomplishing his/her activities and recognize the activities based on the occupant's usage of those objects. For more information about the two activity recognition systems that we have built based on the occupant's usage of everyday objects, we refer to [7], [8].

\subsection{Situative and Multi-modal Interaction Design}

Intelligent environments that provide support to the occupant's activities should consider the occupant's current interactive status before requesting for explicit input or for providing explicit output. Two issues that need to be addressed are [10]: 1) to select the appropriate moment to grab the occupant's attention and interact with him/her since the occupant does not explicitly dedicate all his/her attention, and 2) to

${ }^{1}$ We are interested in activities performed by an individual instead of group activities performed by many occupants in an intelligent environment. 
select the appropriate interactive device to interact with the occupant considering the number and variety of interactive devices present in such environments. The first issue is addressed by considering the action the occupant is currently performing within an activity and based on the status of an activity (initiated, interrupted or completed). For more information about how activities are modelled, we refer to [8]. The second issue is addressed by considering interactive devices around the occupant's body and based on the occupant's perceptual capabilities (explicit output devices within the observable space and explicit input devices within the manipulable space). For more information about observable space and manipulable space which is part of the egocentric interaction model, we refer to [10].

\subsection{Maintaining a Model of the Environment}

Intelligent environments should maintain a real-time model of its environment in a generalized manner required by all its applications. However there is no common way to acquire and maintain a model of the environment. This is a problem addressed within the research area of context-aware computing [11], [12], and [13]. We do not maintain a model of the environment based on absolute location; instead we model the environment in a symbolic manner in terms of the objects that are present in the environment and their relationship to other objects (containment property discussed in [14]) [15]. An important issue that needs to be addressed is to keep track of the objects that enter or leave the intelligent environment in an ad-hoc manner. We classify objects present in an intelligent environment into four types: 1) simple objects - that do not change their internal state like knife, fork, coffee cup, etc; 2) complex objects - that do change their internal state like microwave oven, stove, oven, tap, etc; 3) container objects - that contain other objects like fridge, freezer, cupboard, dining table, etc; and 4) interactive devices - that are used for obtaining explicit input and for providing explicit output like wall mounted display, wrist-worn display, audio headset, speakers, etc. By considering a generalized approach to maintaining a model of the environment, the intelligent environment applications can directly make use of the model without knowing the details of how such information is sensed.

\subsection{Privacy and Personalization}

Intelligent environments should be designed to control the flow of information about its occupants and to personalize the environment based on the occupant's presence, behavior and intentions [16], [17]. However, maintaining a complex model of each person within an intelligent environment is a difficult task to handle, since such models get outdated with time and needs to be updated everytime the occupant enters an intelligent environment. Considering the issues of privacy and personalization, we propose a wearable computing infrastructure for developing and executing intelligent environment applications [10]. However, we are aware of the limitations of a purely wearable computing infrastructure in managing environmental resources among several occupants and in controlling the localized resources in the environment [18]. We intend to address such issues in the future. 


\section{An Activity-Centered Wearable Computing Infrastructure}

Based on the four fundamental challenges described earlier, we present an activitycentered wearable computing infrastructure for developing and executing intelligent environment applications. Refer to Figure 1.

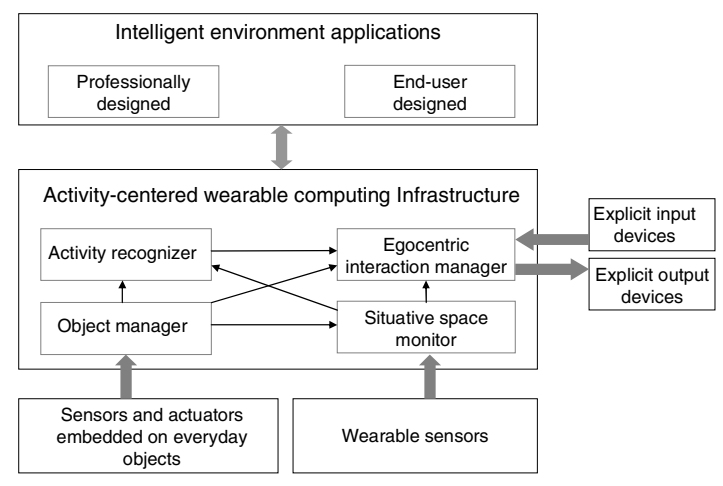

Fig. 1. An activity-centered wearable computing infrastructure for intelligent environment applications, adapted from [10]

Sensors embedded on everyday objects ${ }^{2}$. All everyday objects that are present in the occupant's environment are embedded with passive RFID tags [21]. This includes simple objects, complex objects, container objects and interactive devices. Simple, but multiple state change sensors like on-off switches, temperature sensors, rotation sensors, pressure sensors, etc. are embedded on complex everyday objects to sense their internal states and state changes. RFID readers are embedded on container objects to sense the objects present within the containers and when an object enters or leaves a container [8]. The instrumented sensors communicate with the object manager using ZigBee communication protocol [22].

Wearable sensors. A wearable RFID reader is worn on the inhabitant's chest with three antennas. Two antennas are worn on either wrist to sense the objects grabbed/released events while the third antenna is worn on the chest to sense the objects within the inhabitant's manipulable space [21]. Objects present within the inhabitant's observable space is currently not considered for sensing using real world sensors. However, observable space information channel is used in our VR simulation [7].

Object manager maintains a real-time model of the environment by capturing, storing and managing information about the objects present in the occupant's environment. This component is responsible for the activation or deactivation of the objects that enter or leave the intelligent environment respectively. It stores the activated objects' identity, type, and possible internal states. An object's current

${ }^{2}$ At present, actuators are not embedded on everyday objects and we have left it for future work. 
internal state and current external state in terms of its container object can be queried. For container objects, it stores the identities of the set of objects the container contains. It also stores the event that is generated when an object enters or leaves a container object [8].

- <Activate, \{objectID, Type, “simple”, “complex”, “container”, “interactive”, set_of_internal_states $>>$

- $\quad<$ Deactivate, objectID>

- <objectID, \{current_internal_state $>$

- $\quad<$ objectID, \{containerID $\}>^{3}$

- <containerID, \{set_of_objectIDs\}>

- <objectID, containerID, \{has_entered, has_left $\}>$

Situative space monitor maintains a real-time model of the environment by capturing and storing information about the objects present in the environment from the occupant's egocentric perspective based on what the occupant can see and not see, touch and not touch at any given moment in time [7], [10]. This component stores the set of objects in the occupant's hands, the set of objects within the occupant's manipulable space and the set of objects within the occupant's observable space. It can be queried to check if an object is in the occupant's hands or within the occupant's manipulable space or within the occupant's observable space. It also stores the event that is generated when an object is grabbed or released by the occupant [7].

- $\quad<O M$, \{set_of_objects\} $>$

- $\quad<M S$, \{set_of_objects\} $>$

- $\quad<O S$, \{set_of_objects\}>

- $\quad<$ objectID, OM, $\{$ is_OM $\}>$

- <objectID, MS, \{within_MS\}>

- <objectID, OS, \{within_OS\}>

- $\quad<$ objectID, $\{$ is_grabbed, is_released $\}>$

Egocentric interaction manager is responsible for the situative and multi-modal selection of input and output devices considering the occupant's situation, current activity, personal preferences, and based on the application. It obtains explicit input for the applications and presents explicit output from the applications to the occupant by communicating with the interactive devices that are either worn (using Bluetooth communication) or present in the occupant's environment (using WLAN) [10]. This component is responsible for the activation or deactivation of the devices that are available or not available for interaction respectively. It stores the activated devices' identity, type, access level and modality. It can be queried to obtain the set of available input devices, the set of available input devices within the occupant's manipulable space, the set of available output devices, and the set of available output devices within the occupant's observable space.

- <Activate, \{deviceID, Type, "input”, "output”, AccessLevel, "private”, "public", Modality, "voice", "visual", "gesture”, "tactile" $\}>^{4}$

- $\quad<$ Deactivate, deviceID>

\footnotetext{
${ }^{3}$ containerID is valid only for container objects and is the same as its objectID.

${ }^{4}$ deviceID is valid only for interactive devices and is the same as its objectID.
} 
- <InputDevices, \{set_of_available_input_devices\}>

- $\quad$ InputDevices, $M S$, \{set_of_input_devices_within_MS\}>

- <OutputDevices, \{set_of_available_output_devices\}>

- <OutputDevices, OS, \{set_of_output_devices_within_OS\}>

Activity recognizer is responsible for modeling, recognizing and storing the occupant's activities and actions performed within the intelligent environment. For more information about activity recognition based on object manipulation, manipulable space and observable space information channels, we refer to [7]. For more information about activity recognition based on intra manipulation and extra manipulation information channels, we refer to [8]. This component is responsible for the activation or deactivation of the activities that are included or excluded for recognition respectively. It can be queried for current activity (recognition probability included), current action (recognition probability included), an activity's current status and also an action's current status.

- <Activate, \{activityID, set_of_actionIDs, set_of_mandatory_events $\}>$

- $\quad<$ Deactivate, activityID>

- $<$ CurrentActivity, $\quad$ activityID, activityPR, set_of_actionIDs, set_of_mandatory_events, Status, "initiated", "interrupted", “completed" $\}>$

- <CurrentAction, \{actionID, actionPR, activityID\}>

- $\quad$ activityID, \{activityPR, set_of_actionIDs, set_of_mandatory_events, Status,

"initiated", "interrupted", "completed"\}>

- $\quad$ actionID, \{actionPR, activityID\}>

The object manager, situative space monitor, activity recognizer, egocentric interaction manager and the intelligent environment applications write data into a common black board and exchanges information among themselves using Information and Content Exchange (ICE) Protocol.

\section{Experimental Set-Up and Evaluation}

VR was used as a test-bed [7] in order to speed up the design process of our activitycentered wearable computing infrastructure and to allow us to compensate for the limitations with the currently available sensing technologies (especially for sensing the objects present within the container objects and for sensing the occupant's observable space). A VR model, developed using the Colosseum3D real-time physics platform [19] is used to simulate a physical home environment with wearable sensors and sensors embedded on selected everyday objects (discussed in section 3). Fig. 2 shows a snapshot of our VR simulated intelligent home environment. We have experimented with 78 different objects (128 objects in total) which include 56 different simple objects, 7 different complex objects, 11 different container objects and 4 different interactive devices (wall-mounted display, wrist-worn display, audio headset, and environmental speakers). We represent objects by their object identity (objectID) and not by their individual identity. For instance, both fork_l and fork_2 are represented as fork. Hence all forks have the same objectID. 
The experiments were performed by 5 subjects (none of them are affiliated to the system development team $)^{5}$ in a virtual reality simulated intelligent home environment $^{6}$. 15 activities of daily living were included based on the AMPS framework [20]. The activities were performed 10 times as part of various scenarios. A scenario comprises of a few related activities performed in some sequence. We have used 7 scenarios with some activities common for several scenarios like for instance, the activity of preparing coffee which is common to both the lunch scenario and the coffee-break scenario. All the subjects were allowed to perform the activities in their own way (often in many different ways) within the intelligent home. We had conducted ethnographical studies in 5 households to compare how ADLs are performed in the "real-world" and in the "VR-world". For more information, we refer to [23].

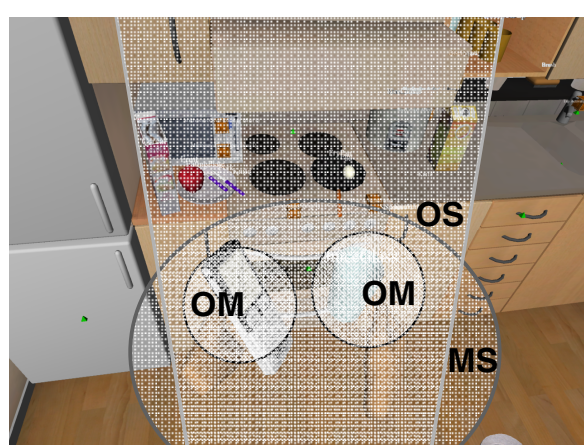

Situative space monitor captures object information within the occupant's OS (observable space), MS (manipulable space) and OM (object manipulation)

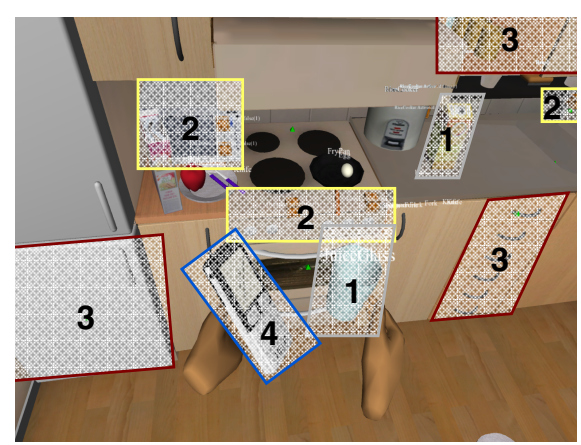

Object manager captures object information available in the occupant's environment: 1) simple object, 2) complex object, 3) container object, and 4) interactive device

Fig. 2. VR simulated intelligent home environment

ADL support for mild-dementia patients. The proposed activity-centered wearable computing infrastructure was evaluated by building a professionally designed ADL support application. To learn about our user group, we have conducted over 10 brainstorming sessions and 4 interview sessions with 6 occupational therapists (from HjälpmedelsCentrum Norr and Geriatric Medicine Department at the University Hospital of Northern Norrland in Sweden).

The data generated based on the activities performed by the 5 subjects were used to train 5 different wearable computing systems, each one personalized for the particular subject. By applying leave-one-out cross-validation (LOOCV) scheme for each subject without mixing the data, we obtain a recognition precision of $92 \%$ at the

${ }^{5}$ Dementia patients were not used as subjects in the VR environment. Based on our initial results, we are currently working on a hardware prototype which will be evaluated by the mild-dementia patients.

${ }^{6}$ The subjects were initially taught how to perform activities in a virtual reality environment and then given a time period to practice in this environment. Only when the subjects were comfortable with the environment, they were allowed to perform the activities. 
activity-level (recall is 99\%) and $81 \%$ at the action-level (recall is 94\%) among 15 ADLs. For more information, refer to [8]. However by mixing the data of all the 5 subjects and applying the LOOCV scheme, we obtain a low recognition precision of $59 \%$ at the activity-level (recall is 92\%) among the 15 ADLs. One important requirement for our application is that the infrastructure should recognize activities and action with high precision and recall values. Hence by considering a personalized approach for modelling and recognizing the occupant's activities, we were able to obtain high precision and recall values.

The support application operates in the occupant's background, constantly keeping track of the activities performed by the occupant. For all the activities initiated by the occupant, the application checks if the occupant has completed the following: 1) Mandatory Events - examples include forgetting to turn off the stove after preparing vegetables, forgetting to place the knife and the fork on the dining table during the activity of preparing the table for lunch, etc., 2) Mandatory Actions - examples include forgetting to clean-up the dining table after having lunch, etc. If the occupant forgets to complete the mandatory events and actions as part of an activity (determined using a sliding window of 2 actions "before" and 2 actions "after" from the system detected current activity state), the application intervenes and attempts to establish communication with the occupant through the egocentric interaction manager.

The egocentric interaction manager was qualitatively evaluated by the 5 subjects. The egocentric interaction manager attempted to grab the occupant's attention by playing a beep sound on his/her Bluetooth headset. Incase the Bluetooth headset is currently not available the system plays the sound in the environmental speakers (if the AccessLevel is "public"). Since mild-dementia patients comprehend better when the events and actions are represented by pictures with less information, we have used pictures with some bright colours that inform about the missing events and actions. The egocentric interaction manager attempts to present this information on a wall-mounted screen present in the occupant's kitchen if the occupant is present in the kitchen environment and if the screen is within or near to his/her observable space. Otherwise this information is presented on the occupant's wrist-worn display. We have also experimented with Voice User Interface (VUI) designed using Dragon Naturally Speaking voice recognition software for obtaining explicit input using voice. Mobile phone keys were used as a complementary option to VUI for obtaining explicit input from the occupant. Even though the egocentric interaction manager at present is based on simple rules, the subjects were positive on the idea of presenting information on different devices based on the occupant's situation. 2 subjects were not completely positive with the idea of having to always wear the Bluetooth headset and the wristworn display for explicitly interacting with the system. In the future, we intend to import more intelligence into the egocentric interaction manager and also experiment with other multimodal interaction approaches that are based on gesture and tactility.

\section{Conclusions}

In this paper we have presented an activity-centered wearable computing infrastructure for developing intelligent environment applications. Four fundamental challenges were outlined for designing intelligent environments and the components 
developed to address such challenges were described and evaluated in a VR simulated home environment. By considering a personalized approach, the activity recognizer has shown as recognition precision of $92 \%$ at the activity-level and $81 \%$ at the actionlevel among 15 ADLs. The egocentric interaction manager has shown initial promise with the idea of interacting with the occupant based on his/her situation within an intelligent environment. An ADL support application capable of providing support for missing mandatory events and actions within activities was developed based on the infrastructure proposed for intelligent environments.

\section{Acknowledgements}

We would like to thank Anders Backman, Björn Sondell, Daniel Sjölie, Fabien Lagriffoul, Gösta Bucht, Kenneth Bodin, Lars-Erik Janlert, and Marcus Maxhall from Umeå University, Sweden. This work is partially funded by the EC Target 1 structural fund program for Northern Norrland, Sweden.

\section{References}

1. Brumitt, B., Meyers, B., Krumm, J., Kern, A., Shafer, S.: EasyLiving: Technologies for Intelligent Environments. In: Proceedings of the Intl. Conf. on Handheld and Ubiquitous Computing, pp. 12-27 (2000)

2. Kidd, C.D., Orr, R.J., Abowd, G.D., Atkeson, C.G., Essa, I.A., MacIntyre, B., Mynatt, E., Starner, T.E., Newstetter, W.: The Aware Home: A Living Laboratory for Ubiquitous Computing Research. In: Streitz, N.A., Hartkopf, V. (eds.) CoBuild 1999. LNCS, vol. 1670, pp. 191-198. Springer, Heidelberg (1999)

3. Mozer, M.C.: The Neural Network House: An Environment that Adapts to its Inhabitants.

In: Coen, M. (ed.) Proceedings of the American Association for Artificial Intelligence Spring Symposium on Intelligent Environments, Menlo Park, CA, pp. 110-114. AAAI Press, Stanford (1998)

4. Backman, A., Bodin, K., Bucht, G., Janlert, L.-E., Maxhall, M., Pederson, T., Sjölie, D., Sondell, B., Surie, D.: easyADL - Wearable Support System for Independent Life despite Dementia. In: Workshop on Designing Technology for People with Cognitive Impairments, CHI2006, pp. 22-23 (April 2006)

5. Coen, M.: Design Principles for Intelligent Environments. In: Proceedings of the 15th national/10th conference on Artificial intelligence/Innovative applications of artificial intelligence (March 23-25, 1998) ISBN:0-262-51098-7

6. Christensen, H.B., Bardram, J.: Supporting Human Activities - Exploring ActivityCentered Computing. In: Borriello, G., Holmquist, L.E. (eds.) UbiComp 2002. LNCS, vol. 2498, pp. 107-116. Springer, Heidelberg (2002)

7. Surie, D., Pederson, T., Lagriffoul, F., Janlert, L.-E., Sjölie, D.: Activity Recognition using an Egocentric Perspective of Everyday Objects. In: UIC 2007. Proceedings of IFIP 2007 International Conference on Ubiquitous Intelligence and Computing. LNCS, vol. 4611, pp. 246-257. Springer, Heidelberg (2007)

8. Surie, D., Lagriffoul, F., Pederson, T., Sjölie, D.: Activity Recognition based on Intra and Extra Manipulation of Everyday Objects. In: UCS2007. Proceedings of the 4th International Symposium on Ubiquitous Computing Systems. LNCS, Springer, Heidelberg (to appear, 2007) 
9. Nardi, B. (ed.): Context and Consciousness: Activity Theory and Human-Computer Interaction. MIT Press, Cambridge (1995)

10. Pederson, T., Surie, D.: Towards an Activity-Aware Wearable Computing Platform based on an Egocentric Interaction Model. In: UCS 2007. Proceedings of the 4th International Symposium on Ubiquitous Computing Systems. LNCS, Springer, Heidelberg (to appear, 2007)

11. Dey, A.: Providing Architectural Support for Building Context-Aware Applications, PhD thesis, College of Computing, Georgia Institute of Technology (December 2000)

12. Dey, A.: Understanding and Using Context. In: Personal and Ubiquitous Computing, vol. 5(1), pp. 4-7. Springer, Heidelberg (2001) ISSN:1617-4909

13. Schmidt, A.: Ubiquitous Computing - Computing in Context, Ph.D. thesis in computer science, Lancaster University (November 2002)

14. Pederson, T.: From Conceptual Links to Causal Relations - Physical-Virtual Artefacts in Mixed-Reality Space. PhD thesis, Dept. of Computing Science, Umeå university, report UMINF-03.14, ISBN 91-7305-556-5 (2003)

15. Hightower, J., Borriello, G.: A Survey and Taxonomy of Location Sensing Systems for Ubiquitous Computing. In: UW CSE 01-08-03, University of Washington, Seattle, WA (August 2001)

16. Langheinrich, M.: Privacy Invasions in Ubiquitous Computing. In: Borriello, G., Holmquist, L.E. (eds.) UbiComp 2002. LNCS, vol. 2498, Springer, Heidelberg (2002)

17. Chatfield, C., Carmichael, D., Hexel, R., Kay, J., Kummerfeld, B.: Personalisation in Intelligent Environments: Managing the Information Flow. In: Proceedings of the 19th conference of the computer-human interaction special interest group (CHISIG) of Australia on Computer-human interaction: citizens online: considerations for today and the future. ACM International Conference Proceeding Series, vol. 122, pp. 1-10 (2005)

18. Rhodes, B., Minar, N., Weaver, J.: Wearable Computing Meets Ubiquitous Computing. The Proceedings of The Third International Symposium on Wearable Computers (ISWC 1999), San Francisco, CA, 141-149 (18-19 October,1999)

19. Backman, A.: Colosseum3D - Authoring Framework for Virtual Environments. In: Proceedings of EUROGRAPHICS Workshop IPT \& EGVE Workshop, pp. 225-226 (2005)

20. AMPS. as on 4nd (September 2007), http://www.ampsintl.com/

21. Finkenzeller, K.: RFID Handbook, 2nd edn. John Wiley and Sons, Chichester (2003)

22. Gutierrez, J.A., Callaway, E.H., Barrett, R.: IEEE: 802.15.4 Low-Rate Wireless Personal Area Networks: Enabling Wireless Sensor Networks, IEEE ISBN-10: 0738135577, ISBN13: 978-0738135571 (November 30, 2003)

23. Bhatt, R.: Comparing the Performance of ADLs in Virtual and Real Life Environments. Dept. of Computing Science, Umeå University, report UMINF-06.40 (2006) 